\title{
Modeling the term structure dynamics in the Nordic electricity swap market
}

\author{
Dennis Frestad, Fred Espen Benth, and Steen Koekebakker
}

\section{Overview}

This paper analyzes the distributions of daily logreturns of financial contracts traded at the Nordic electricity exchange, Nord Pool. These contracts are settled against the average spot price during a delivery period and may accordingly be termed electricity swaps. While fat tailed distributions of electricity spot prices have been investigated by Bottazzi et al. [9], Weron et al. [16], Weron and Misiorek [17], Cartea and Figureroa [11], and Barlow et al. [2], fat-tailed spot prices do not necessarily carry over to electricity swap logreturns, the market invariants essential for derivatives pricing and Value-at-Risk measurement. Due to the non-storability of electricity, Borovkova and Geman [8] claim that "..electricity spot and forward prices are not closely related". Vehviläinen [15] argues likewise that "no analytical connection has been established between the (electricity) spot and forward price." These arguments are equally valid for properly weighted forward prices, swaps. Nevertheless, in an empirical analysis of Nordic electricity swap returns, Benth and Koekebakker [5]

*Corresponding author and conference paper presenter. e-mail address: dennis.frestad@uia.no. Address: University of Agder, Service Box 422, NO-4604 Kristiansand, Norway. 
conclude that a lognormal model of swap dynamics cannot adequately represent the tails of electricity swap return distributions. This suggests that the heavy tails of the electricity spot price carry over to electricity swap returns. The purpose of this study is to analyze the marginal distributions of electricity swap logreturns more carefully across all segments of the Nordic electricity swap market.

Numerous alternative asset return distributions and associated stochastic processes have been put forward to improve risk management and asset pricing practices (Meucci [13], Bouchard and Potters [10], Bibby and Sørensen [7]). During the past decade however, a certain subclass of generalized hyperbolic (GH) distributions has caught momentum following the contributions of Barndorff-Nielsen [[3], [4]], Bibby and Sørensen [6], and Rydberg [14]. A rapidly growing body of evidence demonstrates that the marginal distributions of many stochastic return processes can be successfully fitted by the GH subclass termed normal inverse Gaussian (NIG) distributions. This paper picks up on Benth and Koekebakker's [5] finding that Nordic electricity swap logreturns are heavy tailed and analyzes marginal distributions of daily logreturns in the Nordic electricity swap market from a NIG perspective. It is demonstrated that the NIG distribution provides good fit of electricity swap return distributions in all segments of the electricity market. A stochastic dynamics generating logreturns in correspondence with the fitted NIG distribution, the class of exponential Lévy processes, is also presented. Such processes generalize the more standard geometric Brownian motion in a straightforward way with analytical properties feasible for pricing options and risk management calculations.

The paper is organized as follows. Section 2 introduces the univariate NIG distribution and a useful characterization of the shape of normalized NIG distributions by only two parameters. Section 3 describes the dataset and method used to estimate swap returns in the Nordic electricity market. Section 4 initially presents the 
major empirical findings on shape characteristics, risk premia and volatility in daily swap returns across different swap segments. The quality of the NIG approximation is subsequently analyzed by visual tools for comparative analysis of fit. Finally, a simple illustration highlights the distortions introduced into Value-at-Risk using the normal distribution on fat-tailed electricity swap returns. Section 5 discusses stochastic processes for modeling term structure dynamics and some implications for option pricing. Section 6 concludes.

\section{Methods}

Electricity swap contracts with different delivery periods are traded at the Nordic electricity exchange, Nord Pool. ${ }^{1}$ In order to analyze swap returns in different segments of the Nord Pool market, a smooth curve approximating the prices of traded swap contracts has been constructed for every trading day in the period 1997 to 2005 . Based on the principles of Adams and van Deventer [1], and letting $T \geq t$ denote the delivery time and $t$ the trading date, the smooth curve approximation $\widehat{f}(t, T)$ represents a set of forward prices for different trading dates satisfying the following condition: Swap prices derived from $\widehat{f}(t, T)$ always lie within the corresponding closing price bid-ask spread of their traded counterparts. Difficulties arising from an evolving product structure at Nord Pool are easily overcome with the full set of forward curve approximations, hence a nine-year panel of pseudo-swap prices can be constructed.

With a maximum of two calendar days between trading dates for logreturns, 1750 smooth forward curves $\widehat{f}(t, T)$ are available for analysis. These represent a set of

\footnotetext{
${ }^{1}$ Swaps that are (not) marked-to-market are denoted futures (forwards) by Nord Pool. The term swap denotes both types of derivatives in this paper, a terminology more consistent with the mainstream finance literature (cf. Benth and Koekebakker [5]). More information on this market is available at www.nordpool.com.
} 
latent forward curves $f(t, T)$ capable of reproducing observed swap prices, as well as generating pseudo-prices for non-traded swaps at time $t$. As no dynamic restrictions enter the smoothing algorithm that defines the set of approximations $\widehat{f}(t, T)$, logreturns generated by different swap structures are expected to reveal information about the logreturn generating processes of actual as well as hypothetical swaps. Nevertheless, a swap structure closely resembling traded swaps is selected in this study.

\section{Results}

This paper demonstrates that marginal distributions of daily returns in the Nordic electricity swap market are distinctively non-normal in terms of steepness or tailfatness. It is shown that the flexible class of normal inverse Gaussian distributions captures the observed stylized facts of electricity swap returns accurately across all studied market segments. Furthermore, this family of distributions allows for a stochastic dynamics in terms of Lévy processes, suitable for pricing derivatives and Value-at-Risk measurement.

\section{Conclusions}

Electricity swap returns are distinctively non-normal in terms of steepness or tailfatness, but do not appear to be asymmetric. The NIG family fits empirical electricity swap return distributions very well and represents an attractive alternative to the more familiar location-scale family of normal distributions.

The economic significance of a precise representation of the fat tails of electricity swap returns was demonstrated by a simple Value-at-Risk example. If one is concerned with representing risk exposure with a number that is very unlikely to be 
exceeded, the NIG representation of $V a R$ is clearly superior to $V a R$ calculated under the normal distribution assumption.

Finally, while the marginal distributions of a multivariate NIG distribution are NIG, a multivariate NIG distribution can not easily be inferred from given marginal NIG-specifications and correlations (Lillestøl [12]). Given that some applications have to deal with statistical dependence and therefore require multivariate NIG distributions or the associated copula-function, further research should generalize the findings of this paper into a multivariate NIG framework. 


\section{References}

[1] K. J. Adams and D. R. van Deventer. Fitting yield curves and forward rate curves with maximum smoothness. Journal of Fixed Income, 4(June):55-62, 1994.

[2] M. Barlow, Y. Gusev, and Y. Manpo Lai. Calibration of multifactor models in electricity markets. International Journal of Theoretical and Applied Finance, 7(2):101-120, 2004.

[3] O. E. Barndorff-Nielsen. Normal inverse Gaussian processes and the modelling of stock returns. Technical Report 300, Departement of theoretical statistics, Institute of Mathematics, University of Aarhus, 1995.

[4] O. E. Barndorff-Nielsen. Processes of normal inverse Gaussian type. Finance and Stochastics, 2(1):41, 1998.

[5] F. E. Benth and S. Koekebakker. Stochastic modeling of financial electricity contracts. Forthcoming in Energy Economics.

[6] B. M. Bibby and M. Sørensen. A hyperbolic diffusion model for stock prices. Finance and Stochastics, 1(1):25, 1997.

[7] B. M. Bibby and M. Sørensen. Hyperbolic processes in finance. Technical Report 88, Centre for analytical finance, University of Aarhus and Aarhus school of business, 2001.

[8] S. Borovkova and H. Geman. Analysis and modelling of electricity forward curves. Studies in nonlinear dynamics and econometrics, 10(3):Article 6, 2006. 
[9] G. Bottazzi, S. Sapio, and A. Secchi. Some statistical investigations on the nature and dynamics of electricity prices. Physica A, 355(1):54-61, 2005.

[10] J.-P. Bouchard and M. Potters. Theory of Financial Risk and Derivative Pricing: From Statistical Physics to Risk Management. Cambridge University Press, second edition, 2003.

[11] A. Cartea and M. G. Figueroa. Pricing in electricity markets: A mean reverting jump diffusion model with seasonality. Applied Mathematical Finance, 12(4):313$335,2005$.

[12] J. Lillestøl. Risk analysis and the NIG distribution. Journal of Risk, 2(4):41-56, 2000.

[13] A. Meucci. Risk and asset allocation. Springer Finance. Springer, 2005.

[14] T. Rydberg. The normal inverse Gaussian Lévy process: Simulation and approximation. Communications in statistics - Stochastic models, 13(4):887-910, 1997.

[15] I. Vehviläinen. Basics of electricity derivative pricing in competitive markets. Applied Mathematical Finance, 9(1):45-60, 2002.

[16] R. Weron, M. Bierbrauer, and S. Truck. Modeling electricity prices: jump diffusion and regime switching. Physica A, 336(1/2):39, 2004.

[17] R. Weron and A. Musiorek. Heavy tails and electricity prices: Do time series models with non-Gaussian noise forecast better? Technical Report MPRA Paper No. 2292., Hugo Steinhaus Center for Stochastic Methods. Wroclaw University of Technology, Poland, 2007. 